\title{
Seasonal Variations in the Rupture of Abdominal Aortic Aneurysms
}

\author{
Roberto Manfredini, MD, Francesco Portaluppi, MD, \\ Massimo Gallerani, ${ }^{1} \mathrm{MD}$, Adriana Tassi, MD, \\ Raffaella SALMI, ${ }^{2} \mathrm{MD}$, Paolo ZAMBONI, ${ }^{3} \mathrm{MD}$, \\ Franco Chierici, MD, Savino Occhionoreldi, ${ }^{4} \mathrm{MD}$, \\ Francesco Mascoli, ${ }^{4} \mathrm{MD}$, Emanuela Rizzioli, MD, \\ Alberto LiBONI, ${ }^{3} \mathrm{MD}$, Ippolito DonINI, ${ }^{4} \mathrm{MD}$, \\ and Carmelo Fersini, MD
}

\section{SUMMARY}

From 1982 to 1994,54 patients ( 47 men; mean age 72 years) were referred to the Hospital of Ferrara, Italy for spontaneous rupture of abdominal aortic aneurysm. Sixteen died in the emergency department and 38 underwent urgent surgery. Day and month of onset of acute symptoms leading to urgent surgery were recorded. A seasonal variation with significant peaks in spring and autumn was found.

These findings are likely influenced by local environmental, social and cpidemiological factors, but may be relevant for the appropriate timing of the follow-up and therapeutic strategies for abdominal aortic aneurysms. (Jpn Heart J 1997; 38: 67-72)

Key words: Abdominal aortic aneurysms, Rupture, Natural history, Seasonal variations, Environmental factors

$\mathrm{R}$

ECENT evidence suggests that many unfavorable cardiovascular events occur more frequently during certain hours of the day, and in certain months of the year. Accordingly, a morning peak has been reported in the occurrence of myocardial ischemia ${ }^{1)}$ and infarction, ${ }^{2)}$ sudden cardiac death ${ }^{3)}$, acute pulmonary thromboembolism ${ }^{4)}$, and cerebrovascular diseases. ${ }^{5}$ Moreover, a higher frequency in the winter has also been found for the occurrence of acute cardiovascular ${ }^{6,7)}$ and cerebrovascular diseases. ${ }^{8)}$ Convincing evidence is also

From the Department of Internal Medicine, University of Ferrara, 'Emergency Department, 'Second Medical Division, St. Anna Hospital, Ferrara, ${ }^{3}$ Institute of General Surgery, and ${ }^{4}$ Institute of Clinical Surgery, University of Ferrara, Ferrara, Italy.

Address for correspondence: Roberto Manfredini, MD, Department of Internal Medicine, University of Ferrara, via Savonarola 9, I-44100 Ferrara, Italy.

Supported, in part, by grants from the Italian Ministry of University and Scientific and Technological Research $(60 \%)$.

Received for publication April 8, 1996.

Accepted July 3, 1996. 
available for the presence of both circadian and circannual variations in multiple biologic functions potentially active as triggering factors, e.g., heart rate, blood pressure, coagulation, and fibrinolysis, opening up the potential for a chronopharmacologic approach to cardiovascular diseases.9)

Among acute unfavorable events of the cardiovascular system, the rupture of an abdominal aortic aneurysm (AAA) often plays a dramatic and fatal role. Overall mortality is very high $(80-90 \%)$, and even though most people die before they reach the hospital, $\left.{ }^{10}\right)$ the surgical and intensive care costs of ruptured AAA are a real financial burden to hospitals. ${ }^{11)}$

The possibility of identifying specific or preferred times of higher occurrence of rupture of AAA opens up the potential for targeted diagnostic or interventional strategies. On the other hand, such ruptures may be also influenced by environmental and social factors, and are likely to have populationspecific patterns. Hence, multiple studies in different populations and geographical areas are needed to address this issue.

The purpose of the present study was to investigate whether a seasonal variation exists in the rupture of AAAs in Ferrara, Italy.

\section{Subjects and Methods}

Between January 1982 and December 1994, 54 patients (47 males, 7 females) were referred to the Hospital of Ferrara, Italy, for spontaneous rupture of an AAA. Of these, 16 patients died in the Emergency Department (ED) before surgery could be performed and 38 had emergency surgery. The diagnosis of ruptured AAA was based on the presence at laparatomy of a retroperitoneal haematoma or free blood in the peritoneal cavity. In all of the cases who died in the $\mathrm{ED}$, diagnosis was confirmed by autopsy. Day and month of occurrence of the acute event were recorded.

Group comparisons were assessed using Wilcoxon's test or, when applicable, one-way analysis of variance after checking homogeneity of variances with Bartlett's test. Student-Newman-Keuls multiple comparison test was used as a post-hoc procedure. Temporal distribution was analysed both after grouping all cases by season (3-month intervals) and after categorizing all cases into twelve 1month periods. First, a chi-squared test for goodness of fit was applied to the number of observed versus expected ruptured AAA during four 3-month intervals. Then, analysis of variance was calculated. Finally, rhythm analysis was performed by fitting partial Fourier series with up to 5 harmonics to the data. The significance level for all data was $p<0.05$. 


\section{Results}

Fifty-four cases were included in this study, with a clear preponderance of males (male to female ratio $=7: 1$ ). The demographic features of the study population are reported in the Table 1 . In the total population studied, the mean age was 72 years. However, mean age was higher in the group who died in the ED as compared to those who underwent surgery $(p<0.0001)$. In addition, male patients were significantly younger than females $(69 \pm 7.4$ vs $79 \pm 6.7$ years, $p=0.014$, and $76 \pm 4.2$ vs $85 \pm 7.8$ years, $p=0.012$, respectively), indicating a more rapid progression toward rupture of AAA in men.

The monthly distribution of hospital admissions for AAA is reported in Figure 1. After grouping the cases by season, significant spring and autumn peaks were found for ruptured $(p=0.026)$. Analysis of variance demonstrated a variation significantly greater than expected by chance $(p=0.028)$. Rhythm analysis of monthly distribution demonstrated a significant pattern, characterized by a 6 -

Table. Distribution of Abdominal Aortic Aneurysms According to Urgency, Sex and Age

\begin{tabular}{crrc}
\hline & $n$ & $\%$ & men age \pm SD \\
\hline Total subjects & 54 & 100.0 & $72 \pm 8.0$ \\
Males & 47 & 87.0 & $71 \pm 9.0$ \\
Females & 7 & 13.0 & $81 \pm 7.0$ \\
Death in the ED & 16 & 12.4 & $77 \pm 6.0$ \\
Males & 13 & 10.1 & $76 \pm 4.2$ \\
Females & 3 & 2.3 & $85 \pm 7.8$ \\
Urgent Surgery & 38 & 29.5 & $70 \pm 8.0$ \\
Males & 34 & 26.4 & $69 \pm 7.4$ \\
Females & 4 & 3.1 & $79 \pm 6.7$ \\
\hline
\end{tabular}

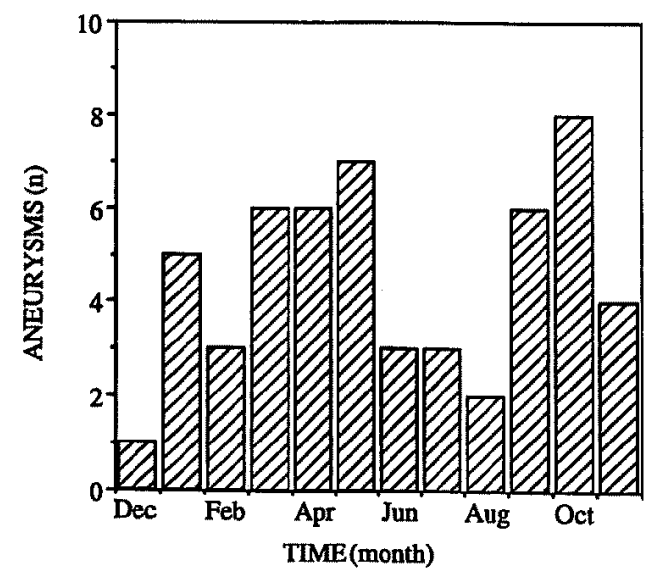

Figure 1. Monthly distribution of ruptured abdominal aortic aneurysms (raw data on cartesian axes). 


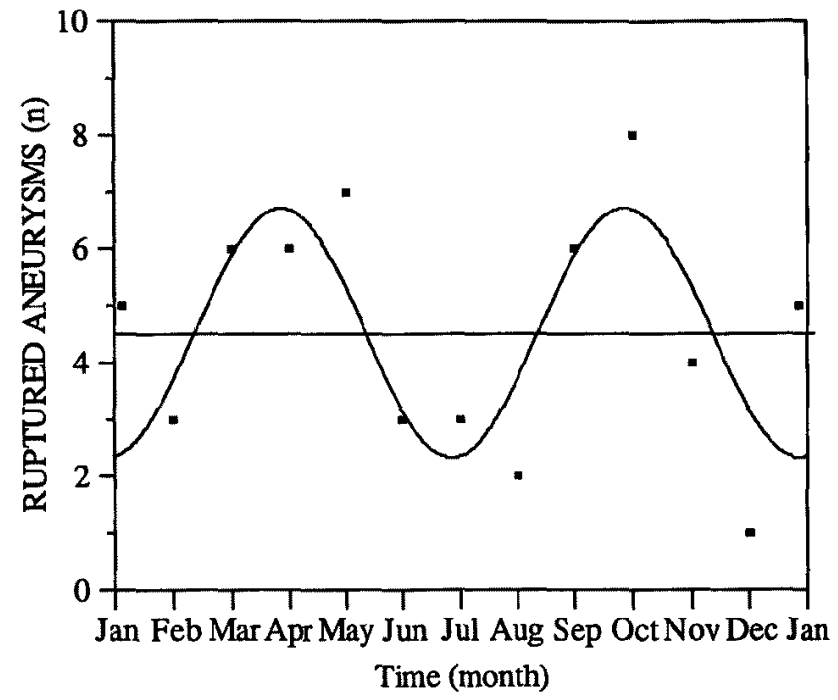

Figure 2. Monthly distribution of ruptured abdominal aortic aneurysms (rhythmometric analysis by fitting partial Fourier series).

month period with two peaks occurring in April and October $(p=0.024)$, respectively (Figure 2).

\section{Discussion}

The main finding of this study was the demonstration of a peculiar seasonal pattern of occurrence of ruptured AAA in northeastern Italy, with two significant peaks in spring and autumn, confirming a recent observation in Great Britain on a sample collected from 1987 to $1991 .^{12)}$

Two previous studies were conducted in Western Australia ${ }^{13)}$ (376 cases over an 11-year study) and in Greece ${ }^{14)}$ (66 cases over 23 years): both identified seasonal patterns in rupture of AAA, but with a higher frequency in winter and autumn, respectively. Many relevant variables, such as those related to climate, socioeconomic conditions, anagraphic composition, and risk factor distribution, may greatly differ among nations, making conclusions drawn from observational studies population-specific.

The causes of spontaneous rupture of AAA are still incompletely understood, but they are likely to reside in an imbalance among multiple interactive factors of arterial wall integrity, leading to a weakened aortic wall and/or an increased load upon it. ${ }^{15)}$

Hypertension is associated with AA rupture ${ }^{16)}$, although its pathogenetic role has not been demonstrated. 
A seasonal influence on blood pressure is known, with higher blood pressures in winter than in summer, and greater seasonal variations in older than in younger subjects. ${ }^{17)}$ Thus, it is commonly accepted that environmental temperature has an inverse relation with blood pressure. ${ }^{18)}$ During peripheral vasoconstriction, systolic blood pressure increases more than diastolic, so that pulse amplitude is generally increased, although there is a little change in cardiac output or pulse rate. ${ }^{19)}$ This enhances the forces acting to produce wall deformation and increase friction and shear stress on the internal surface. The consequent vascular damage may progress until arterial dissection and rupture of the aorta are determined ${ }^{27}$ ) Such mechanisms, undoubtedly present in north-eastern Italy during the colder months, could be activated and exacerbated in this geographical area also in the months of transitional seasons, October-November but also MarchApril, when multiple and frequent stress episodes on the arterial wall caused by the typical temperature excursions of these time periods may precipitate aneurysmal rupture more than the more stable (but not rigid) winter cold.

The demonstration of a seasonal variation in the occurrence of ruptured AAA might have therapeutic implications. In addition to the widely accepted clinical and instrumental criteria, consideration of the time of the year might prove useful in the making of the decision to intervene surgically.

In conclusion, the demonstration of a seasonal pattern of rupture may be a useful complement to our knowledge of the natural history of abdominal aortic aneurysms. The follow-up strategies and the indication for elective surgery might be improved if appropriately timed to the periods of the year of higher risk of rupture in each population.

\section{ReFERENGES}

1. Nademanee K, Intarachot V, Josephson MA, Singh BN. Circadian variation in occurrence of transient overt and silent myocardial ischemia in chronic stable angina and comparison with Prinzmetal angina in man. Am J Cardiol 1987; 60: 494-8.

2. Muller JE, Stone PH, Turi ZG, ct al. Circadian variation in the frequency of onset of acute myocardial infarction. N Engl J Med 1985; 313: 1315-22.

3. Muller JE, Ludmer PL, Willich SN, et al. Circadian variation in the frequency of sudden cardiac death. Circulation 1987; 75: 131-8.

4. Gallerani M, Manfredini $R$, Ricci L, et al. Sudden death from pulmonary thromboembolism: chronobiological aspects. Eur Heart J 1992; 13: 661-5.

5. Gallerani M, Manfredini R, Ricci L, et al. Chronobiological aspects of acute cerebrovascular diseases. Acta Neurol Scand 1993; 87: 482-7.

6. Protos AA, Caracta A, Gross L. The seasonal susceptibility to myocardial infarction.J Am Geriatr Soc $1971 ; 19: 526-35$.

7. Manfredini R, Gallerani M, Salmi R, Zamboni P, Fersini C. Fatal pulmonary embolism in hospitalized patients: evidence for a winter peak. J Int Med Res 1994; 22: 85-9.

8. Gallerani M, Trapella G, Manfredini R, Pasin M, Napolitano M, Migliorc A. Acute intracerebral haemorrhage: circadian and circannual patterns of onset. Acta Neurol Scand 1994; 89: 280-6. 
9. Manfredini R, Gallerani M, Salmi R, Fersini C. Circadian rhythms and the heart: implications for chronotherapy of cardiovascular diseases. Clin Pharmacol Ther 1994; 56: $244-7$.

10. Smith R. Britain needs to screen for aortic aneurysms. Br Med J 1994; 308: 358-9.

11. Breckwoldt WL, Mackey WC, O'Donnel TF, The economic implications of high-risk abdominal aortic aneurysms. J Vasc Surg 1991;13: 798-804.

12. Varty K, Reid A, Jagger G, Bell PRF. Vascular emergencies: what's in season? Cardiovasc Surg 1995; 3: 409-11.

13. Castleden WM, Mercer JC. Abdominal aortic aneurysms in Western Australia: descriptive epidemiology and patterns of rupture. Br J Surg 1985; 72: 109-12.

14. Liapis $\mathrm{C}$, Sechas $\mathrm{M}$, niopoulos $\mathrm{D}$, et al. Seasonal variation in the incidence of ruptured abdominal aortic aneurysm. Eur J Vasc Surg 1992; 6: 416-8.

15. MacSweeney STR, Powell JT, Greenhalgh RM. Pathogenesis of abdominal aortic aneurysm. Br J Surg 1994; 81: 935 41.

16. Spittel JA Jr. Hypertension and arterial aneurysm. J Am Coll Cardiol 1983; 1: 533-40.

17. Brennan PJ, Greenberg G, Miall WE, Thompson SG. Seasonal variation in arterial blood pressure. Br Med J 1982; 285: 91923.

18. Kunes J, Tremblay J, Bellavancé $\mathbf{F}$, Hamet $\mathbf{P}$. Influence of environmental temperature on the blood pressure of hypertensive patients in Montreal. Am J Hypertens 1991; 4: 422-6.

19. Keatinge WR, Coleshaw SR, Cotter F, Mattock M, Murphy M, Chelliah R. Increases in platelet and red cell counts, blood viscosity, and arterial pressure during mild surface cooling: factors in mortality from coronary and cerebral thrombosis in winter. Br Med J 1984; 289: 1405-8.

20. Wilmshurst $P$. Temperature and cardiovascular mortality. Br Med J 1994; 309: 1029-30. 